ОСОБЕННОСТИ КОСТНОГО МЕТАБОЛИЗМА ПРИ САХАРНОМ ДИАБЕТЕ

(с) Г.М. Нуруллина, Г.И. Ахмадуллина

ФГБОУ ВО "Ижевская государственная медицинская академия" Минздрава России, Ижевск, Россия

Пациенты с сахарным диабетом (СД) имеют повышенный риск развития остеопоротических переломов, что связано со снижением прочности кости.

Накопление конечных продуктов гликирования, гипергомоцистеинемия увеличивают апоптоз остеоцитов, уменьшают костеобразование и замедляют костное ремоделирование, при СД. Адипонектин стимулирует экспрессию остеокальцина и дифференцировку остеобластов посредством активации 5'АМФ-активируемой протеинкиназы (АМФК). АМФК-активация стимулирует дифференцировку и минерализацию остеобластов. Гипоадипонектинемия, которая часто наблюдается при ожирении и диабете, может участвовать в снижении прочности кости при СД.

Замедленный костный метаболизм у пациентов сахарным диабетом подтверждается при изучении уровня маркеров костного ремоделирования. Об этом свидетельствует снижение уровня маркеров костного образования (остеокальцин, P1NP), маркеров костной резорбции (CTX, TRAP), повышение маркеров-регуляторов костного обмена (OPG, CKлеростин).

Таким образом, изучение патофизиологии костного метаболизма, уровня маркеров костного обмена у пациентов с сахарным диабетом дает широкие перспективы в понимании механизмов развития остеопороза как осложнения сахарного диабета, подбора таргетной терапии и улучшения ранней диагностики заболевания.

КЛЮЧЕВЫЕ СЛОВА: Сахарный диабет 2 типа; остеопороз; особенности костного метаболизма.

\title{
FEATURES OF BONE TURNOVER IN DIABETES MELLITUS
}

(c) Guzel M. Nurullina, Guzyal I. Akhmadullina

Izhevsk State Medical Academy, Izhevsk, Russia

Patients with diabetes mellitus (DM) have an increased risk of osteoporotic fractures, which is associated with a bone fragility.

Accumulation of advanced glycation end products, hyperhomocysteinemia causes increased apoptosis of osteocytes, decreased bone formation and bone remodeling in DM. Adiponectin stimulates osteocalcin expression and osteoblast differentiation through the activation of AMPK. AMPK-activation stimulates differentiation and mineralization of osteoblasts. Hypoadiponectinemia, which is often observed in obesity and diabetes, can causes bone fragility.

Diabetes mellitus is a state of low bone turnover, which is confirmed by decreased markers of bone formation (osteocalcin, P1NP), decreased markers of bone resorption (CTX, TRAP), increased regulatory markers of bone remodeling (OPG, sclerostin).

Thus, the study of the pathophysiology of bone metabolism, the level of bone metabolism markers in patients with diabetes mellitus gives broad prospects in understanding the mechanisms of osteoporosis as complication of diabetes mellitus, the selection of targeted therapy and the improvement of early diagnosis of the disease.

KEYWORDS: Diabetes mellitus; osteoporosis; bone turnover.

\section{ВВЕДЕНИЕ}

Остеопороз и сахарный диабет имеют на сегодняшний день высокую медико-социальную значимость, что обусловлено высокой распространенностью данных заболеваний, снижением качества жизни пациентов, повышением инвалидизации и смертности.

Длительное время сахарный диабет и остеопороз рассматривались как отдельные заболевания. Предыдущие исследования показали, что пациенты с сахарным диабетом 1 типа (СД1) и 2 типа (СД2) имеют повышенный риск развития остеопоротических переломов [1-3]. Поэтому остеопороз в последнее время считается одним из диабетических осложнений в связи со снижением прочности кости, развивающимся на фоне сахарного диабета. Так, по данным метаанализа 2007 года пациенты с СД1 типа имели более низкую минеральную плотность костной ткани (МПК) поясничного отдела позвоночника и бедра (Z-критерий-0.22SD и -0.37SD, соответственно), у пациентов с СД2 были более высокие показатели МПК поясничного отдела позвоночника и бедра (Z-критерий $+0,41 \mathrm{SD}$ и +0,27SD, соответственно). Согласно значениям МПК, оценочные риски переломов имели 1,42-кратное увеличение при СД1 и 0,77-кратное уменьшение при СД2. Однако риск перелома шейки бедра по сравнению с контролем без диабета были увеличен в 6,9 раз 
при СД1 и в 1,4 раза при СД2 [1]. Более того, другой метаанализ 2007 года также показал, что риск перелома шейки бедра для пациентов с СД1 и СД2 был увеличен до 6,3 и 1,7 раза соответственно, по сравнению с контролем без диабета [2]. Кроме того, наличие СД2 является независимым фактором риска развития переломов позвонков после корректировки на возраст, индекс массы тела и МПК позвоночника [3]. Таким образом, снижение прочности кости, обусловленное сахарным диабетом, вызвано, главным образом, ухудшением качества кости, а не уменьшением МПК, поэтому измерение МПК у пациентов с диабетом может быть менее информативным, чем для пациентов без диабета [4]. Соответственно, изучение патофизиологии костного метаболизма, уровня маркеров костного обмена у пациентов с сахарным диабетом дает широкие перспективы в понимании механизмов развития остеопороза как осложнения сахарного диабета, подбора таргетной терапии и улучшения ранней диагностики заболевания.

\section{ПАТОГЕНЕЗ НАРУШЕНИЙ КОСТНОГО ОБМЕНА ПРИ САХАРНОМ ДИАБЕТЕ}

\section{Конечные продукты гликозилирования}

Важный фактор, который может способствовать снижению прочности кости при сахарном диабете 2 типа посттрансляционное гликирование коллагена в костном матриксе. Ферментативные сшивки коллагена поддерживают прочность нормального костного матрикса, коллагеновая матрица придает пластичность, позволяя кости выдержать деформацию без разрушения. Воздействие высокого уровня глюкозы способствует накоплению конечных продуктов гликирования (КПГ, advanced glycation end products, AGEs]. В коллагене КПГ приводят к образованию неферментативных поперечных сшивок. Увеличение числа поперечных сшивок коллагена уменьшает пластичность матрицы, и кость разрушается при деформации [5]. Считается, что КПГ в настоящее время играют решающую роль в развитии диабетических осложнений, потому что гипергликемия и окислительный стресс ускоряют формирование КПГ [4].

Предыдущие исследования показали, что накопление КПГ в кости приводит к МПК-независимому снижению прочности кости у пациентов с диабетом. Известно, что КПГ накапливаются с возрастом в различных тканях, включая кость, почки, мозг и атеросклеротические бляшки коронарных артерий. В нескольких исследованиях было показано, что уровни сывороточного КПГ были значительно выше у пациентов с диабетом, чем у здоровых людей. Среди КПГ пентозидин является хорошо изученным соединением и считается достоверным предиктором развития микро- и макрососудистых осложнений у пациентов с диабетом [6].

Saito et al. ранее продемонстрировали, что у крыс с диабетом наблюдалось значительное увеличение пентозидиновых коллагеновых сшивок в кости, которое ассоциировалось с ухудшением механических свойств кости, несмотря на нормальную костную массу [7]. Schwartz et al. ранее продемонстрировал, что более высокий уровень пентозидина мочи достоверно связан с увеличением частоты переломов у пожилых пациентов с СД2 в когортном исследовании [8]. Кроме того, ранее в кросс-секционном исследовании было доказано, что уровни пентазидина в сыворотке достоверно и положительно ассоциируются с распространенными при СД2 переломами позвоночника у женщин в постменопаузе [9]. Недавнее клиническое исследование, в котором проводилась биопсия кости у пациентов с СД1 показало, что содержание пентозидина в трабекулах было достоверно и положительно связано с уровнем $\mathrm{HbA}_{1 c^{\prime}}$ и было увеличено у СД1 пациентов с переломами [10].

Прочность материала кости можно оценить in vivo с помощью Osteoprobe. OsteoProbe ${ }^{\circledR}$ - это портативный карманный микроиндентор, который измеряет способность костного материала сопротивляться вдавливанию. Прочность костной ткани, исследованной с помощью OsteoProbe, при сахарном диабете 2 типа ниже на 10\%, чем у контроля. Разница сохраняется после коррекции на ИМТ и коррелирует со средним значением $\mathrm{HbA}_{1 c}$ [11]. Непрямое измерение КПГ с использованием автоматической флуоресценции кожи объясняет снижение прочности кости на $26 \%$ при применении метода вдавливания и более низкий P1NP у пациентов с СД2 [12]. Следовательно, есть доказательства наличия взаимосвязи повышенного уровня глюкозы с низким качеством костной ткани при СД2 и гликирование коллагена является фактором повышения риска перелома.

Конечные продукты гликозилирования непосредственно влияют на клетки костного образования. Рецептор для КПГ (RAGE) экспрессируется остеобластами и остеоцитами [13], и гипергликемия увеличивает экспрессию RAGE [14]. В ранее проведенных исследованиях было установлено, что сочетание высокого уровня глюкозы и КПГ и нгибирует минерализацию в клеточных линиях предшественников остеобластов МС3Т3-E1 [14], и КПГ ингибирует остеобластную дифференцировку или минерализацию стромальных ST2 клеток мышей и человеческих мезенхимальных стволовых клеток путем уменьшения экспрессии остерикса, увеличения трансформирующего ростового фактора (TGF)- $\beta$ и подавления стрессовых белков эндоплазматического ретикулума $[15,16]$ (рис. 1). Более того, гипергликемия и КПГ достоверно увеличивают экспрессию склеростина в остеоцитоподобных MLO-Y4 клетках [13]. Напротив, КПГ уменьшают экспрессию RANKL, который стимулирует дифференцировку и активность остеокластов. Кроме того, КПГ индуцируют апоптоз остеобластов и остеоцитов $[13,15]$. Вместе взятые, эти данные свидетельствуют о том, что гипергликемия и КПГ ингибируют дифференцировку остеобластов и образование костной ткани напрямую и косвенно путем увеличения экспрессии склеростина в остеоцитах, а также способствуют низкому костному ремоделированию, уменьшая экспрессию RANKL в остеоцитах.

Считается, что подавление ремоделирования кости способствует снижению прочности костной ткани у пациентов с СД. Костная ткань постоянно обновляется с помощью баланса между образованием кости остеобластами и остеокластической резорбцией кости. Таким образом, когда процесс ремоделирования кости нарушен, старая костная ткань, имеющая коллагеновые сшивки из-за КПГ, не будет обновляться, что приведет к ухудшению качества кости. 


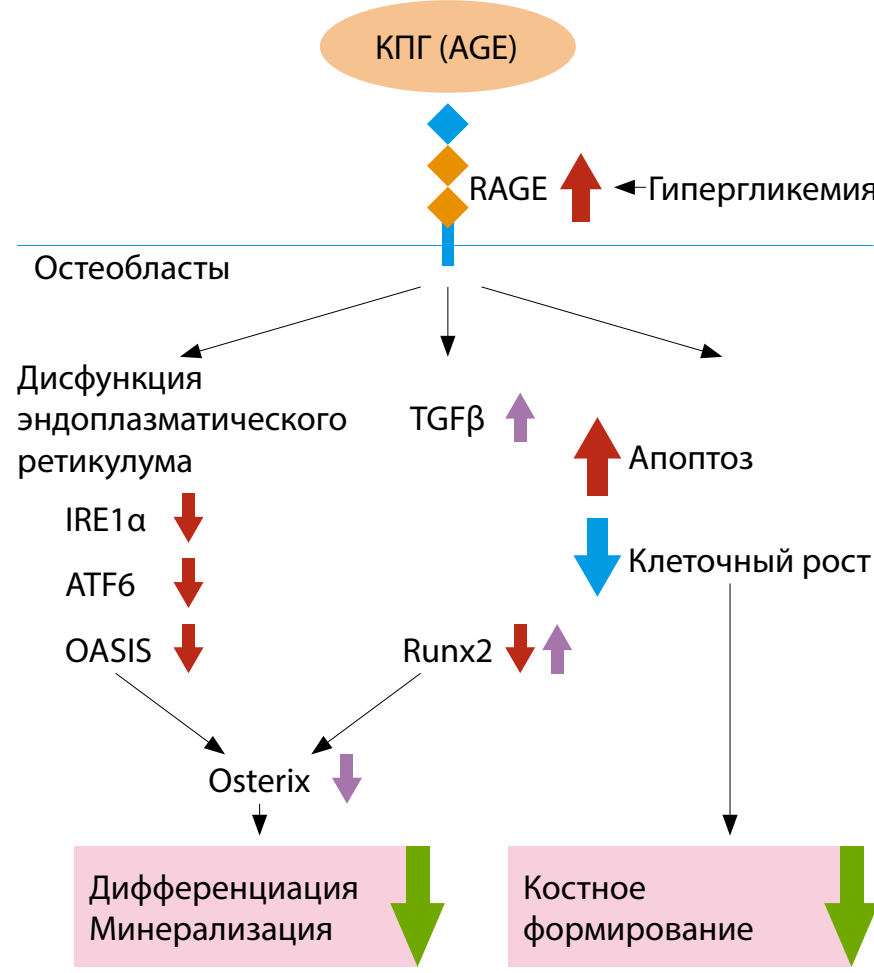

Рис. 1. Рецептор для AGE (RAGE) экспрессируется на остеобластах. Гипергликемия увеличивает экспрессию (RAGE). КПГ индуцирует апоптоз остеобластов и подавляет рост остеобластов. КПГ ингибирует дифференциацию и минерализацию остеобластов посредством дисфункции эндоплазматического ретикулума и экспрессии трансформирующего фактора роста $\beta$ (TGF $\beta$ ), приводя к снижению костного образования.

IRE1a - инозитол регулирующий энзим 1a. ATF6 - активирующий фактор транскрипции 6

\section{Гомоцистеин}

Гомоцистеин (Нсу) представляет собой серосодержащую аминокислоту, образованную деметилированием метионина, и высокие уровни Нсу плазмы вызваны старением, заболеваниями, связанными с образом жизни, такими, как диабет, а также витамин В12 - и фолатная недостаточность. Предыдущие исследования показали, что гипергомоцистеинемия увеличивает риск развития остеопоротических переломов независимо от МПК $[17,18]$, предполагая, что ухудшение качества кости может быть доминирующей причиной Нсу -индуцированной хрупкости костей. Li et al. ранее сообщили, что уровни Нсу плазмы были достоверно выше у пациентов с СД2 по сравнению с пациентами, не страдающими диабетом, и что более высокие уровни Нсу были связаны с распространенностью переломов позвонков и бедра у пациентов с СД2 [19]. Диет-индуцированная гипергомоцистеинемия снижает качество костной ткани in vivo [20], и Нсу непосредственно поражает клеточные линии остеобластов, такие как стромальные клетки костного мозга и остеобласты. Нсу индуцирует апоптоз остеобластов путем увеличения окислительного стресса [21]. Помимо этого, Нсу подавляет экспрессию лизилоксидазы, которая образует поперечные связи коллагеновых фибрилл, увеличивает накопление внеклеточного пентозидина в остеобластах. Эти данные свидетельствуют о том, что Нсу может ухудшить жизнеспособность и функцию остеобластов, а также ухудшить жесткость костной ткани путем ингибирования образования ферментативных коллагеновых сшивок и увеличения внеклеточных неферментативных пентозидиновых сшивок (рис. 2). Более
Гомоцистеин

$\downarrow$

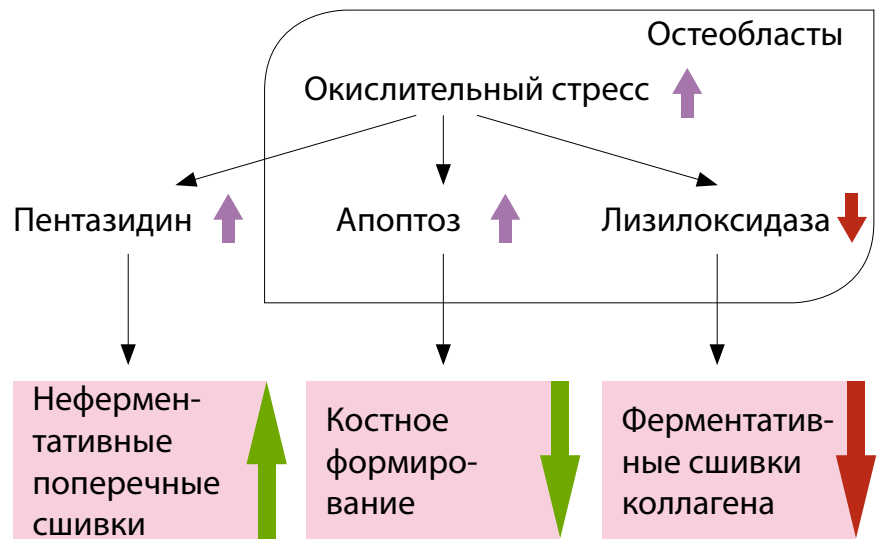

Рис. 2. Нсу (гомоцистеин) увеличивает внутриклеточный оксидативный стресс в остеобластах и индуцирует апоптоз. Нсу подавляет экспрессию лизилоксидазы, которая является наиболее важным ферментом для формирования коллагеновых поперечных связей и увеличивает внеклеточное накопление пентозидина

того, Нсу усиливает окислительный стресс и индуцирует апоптоз остеоцитов путем увеличения NADPH оксидазы 1 (Nox1) и Nox2 [22, 23]. Эти данные свидетельствуют о том, что Нсу индуцирует дисфункцию остеобластов и накопление КПГ и увеличивает апоптоз остеоцитов, что приводит к уменьшению образования и ремоделирования костей, а также увеличению КПГ-коллагеновых сшивок. Поэтому не только КПГ, но и гипергомоцистеинемия может быть вовлечена в снижение прочности костной ткани при сахарном диабете.

Инсулин и инсулиноподобный фактор роста-1

Пациенты с СД1 имеют значительное снижение МПК с уменьшением костеобразования и повышенный риск остеопоротических переломов [24].

Эта клиническая особенность предполагает, что необходим сигнал инсулина для формирования костной ткани и их развития. Предыдущие исследования показали, что остеобласты имеют функциональные инсулиновые рецепторы (Ob-IR) и что лечение инсулином стимулирует пролиферацию и дифференциацию остеобластов [25]. Исследования, проведенные на мышах, нокаутных по остеобласт-специфическому инсулиновому рецептору (Ob-IR - / -), выявили снижение объема кости из-за уменьшения образования костей и дефицита остеобластов [26]. Инсулин подавляет ингибитор Runx2 Twist2, что способствует остеобластной дифференцировке, необходимой для нормального формирования кости [26]. Инсулиноподобный фактор роста (ИФР-1) известен анаболическим влиянием на кость. ИФР-1 экспрессируется в остеобластах и аутокринно и паракринно в микроокружении влияет на дифференцировку остеобластов и ремоделирование кости. Предыдущие исследования выявили, что нокаутные мыши по рецептору ИРФ-1 имели достоверное снижение массы костной ткани и недостаточную минерализацию [27], у ИРФ-1 - нулевых мышей наблюдалось заметное уменьшение костного объема, периостальной окружности и медиальной боковой ширины [28]. Кроме того, несколько лабораторных исследований показали, что стимулирующее влияние 
ИРФ-1 на остеобласты ингибируется гипергликемией и КПГ [29, 30], и что высокий уровень глюкозы значительно ухудшает пролиферативную и функциональные реакции остеобластов на ИРФ-1 [29]. КПГ значительно уменьшают секрецию ИРФ-1 в остеобластах [30]. уровень ИРФ-1 в сыворотке положительно коррелирует с уровнями остеокальцина в сыворотке, и обратно пропорционально числу переломов позвонков у женщин в постменопаузе с СД2 [31]. Таким образом, гипергликемия и КПГ могут вызывать резистентность остеобластов к ИРФ-1 влиянию в локальной среде. Следовательно, сигнализация ИРФ-1 важна для поддержания костной массы и прочности кости у пациентов с диабетом, а также снижение уровней ИРФ-1 может участвовать в развитии хрупкости кости, обусловленной СД.

\section{Адипонектин}

Предыдущие исследования показали, что жировая ткань связана с метаболизмом костной ткани. Провоспалительные цитокины висцерального жира, такие как, интерлейкин-6 (IL-6) и фактор некроза опухолей альфа (TNF-a) повышают костную резорбцию. [32].

Адипоциты секретируют различные биологически активные молекулы, которые называются адипокинами. Было показано, что остеобласты имеют адипонектиновые рецепторы, адипонектиновая сигнализация стимулирует пролиферацию, дифференциацию и минерализацию остеобластов $[33,34]$. Ранеe Luo et et al. показали, что рекомбинантный адипонектин усиливает активность ALP и экспрессию остеокальцина в остеобластах человека [34]. Кроме того, адипонектин активирует АМФК и стимулирует дифференцировку и минерализацию остеобластов путем увеличения костного морфогенетического белка-2 (ВМР-2). Нокдаун (разрушение) рецептора адипонектина с использованием siRNA ингибирует активность ALP, а также экспрессию остеокальцина в остеобластических клетках МС3Т3-E1 [33]. Более того, также сообщается, что адипонектин стимулирует активность остеокластов, увеличивая экспрессию RANKL и уменьшая экспрессию OPG в остеобластах, хотя адипонектин не оказывает прямого воздействия на остеокласты [35]. Эти выводы дают возможность предполагать, что адипонектин играет важную роль в костеобразовании и ремоделировании, и что гипоадипонектинемия, которая часто наблюдается при ожирении и диабете у пациентов, может участвовать в снижении прочности кости при СД. Однако связь между адипонектином и костным метаболизмом до сих пор неясна in vivo и в клинических исследованиях. Несколько исследований показали, что уровень адипонектина положительно коррелировал с маркерами костеобразования $[36,37]$, тогда как ряд других исследований показали, что более высокий уровень адипонектина в сыворотке связан с риском переломов $[36,38]$. Поэтому необходимы дальнейшие исследования для изучения влияния адипонектина на метаболизм костей и риск перелома у пациентов с СД.

Роль АМФК в костном метаболизме и взаимодействии между костным и углеводным метаболизмом

AMФК (5'АМФ-активируемая протеинкиназа, АMP activated protein kinase, AMPK), как известно, является решающим регулятором энергетического и метаболи- ческого гомеостаза на клеточном уровне и уровне организма [39]. Увеличение в клетке соотношения АМФ/АТФ активирует АМФК через фосфорилирование а-субъединицы. После активации, АМФК инактивирует несколько метаболических ферментов, вовлеченных в клеточные процессы с использованием АТФ, включая синтез холестерина и белка [40]. Накопленные доказательства показали, что АМФК является кандидатом для таргетной терапии заболеваний, связанных с нарушением метаболизма, атеросклероза и рака [41]. Субъединицы АМФК экспрессируются в костной ткани и других клетках; а1-субъединица АМФК является доминирующей каталитической изоформой, экспрессирующейся в костях [42]. Предыдущие исследования продемонстрировали, что передача сигналов АМФК играет важную роль в костной физиологии [43]. АМФК-активация стимулирует дифференцировку и минерализацию остеобластов, усиливая экспрессию BMP-2 и эндотелиальную синтазу оксида азота (eNOS, endothelial nitric oxide synthase) через ингибирование мевалонатного пути $[33,44]$. Более того, другие исследователи также сообщили, что ингибирование АМФК подавляло остеобластную дифференцировку [45]. С другой стороны, несколько исследований показало, что активация АМФК напрямую ингибирует остеокластогенез [46] и опосредованно подавляет дифференцировку остеокластов путем стимуляции OPG и снижения экспрессии RANKL в остеобластах [47]. Более того, активация AMФК достоверно снижает экспрессию RANKL и увеличивает экспрессию склеростина в остеоцитах MLO-Y4 клеток, и нокдаун АМФКа1 достоверно увеличивает экспрессию RANKL [48]. Вместе эти данные свидетельствуют о том, что активация АМФК в остеобластах стимулирует их дифференцировку и минерализацию, но активация АМФК в остеоцитах подавляет дифференцировку остеобластов путем увеличения экспрессии склеростина, возможно, по принципу отрицательной обратной связи. В противоположность этому, АМФК-активация в остеобластах и остеоцитах ингибирует дифференцировку остеокластов путем уменьшения экспрессии RANKL и стимулирования экспрессии OPG, и активация АМФК в остеокластах подавляет их дифференцировку и активность. Действительно, несколько исследований, проведенных на генетически измененных мышах, показали, что инактивация АМФК уменьшает костную массу in vivo $[41,43,48]$. Исследование нокаутных мышей по а-субъединице АМФК показало, что компартментов кортикальной и трабекулярной кости у них было достоверно меньше по сравнению с контролем из-за повышенной резорбции кости [48]. Как сообщалось ранее, остеобласты имеют функциональный адипонектиновый рецептор и адипонектин увеличивает дифференцировку остеобластов и ремоделирование кости через АМФК-сигнализацию. Кроме того, адипонектин и АМФК-активация стимулирует экспрессию остеокальцина в остеобластах [33]. Адипонектин стимулирует экспрессию остеокальцина в остеобластах и дифференцировку остеокластов, и остеокальцин также стимулирует экспрессию адипонектина в адипоцитах; поэтому разумно предположить, что существует эндокринная связь между костной и жировой тканью через активацию АМФК в кости.

С другой стороны, сообщается, что ИРФ-1 модулирует АМФК в различных типах клеток [49]. Более того, 
недавнее исследование показало, что АМФК-активация необходима для активации ИРФ-1 на ранних стадиях дифференцировки остеобластов [50]. Поэтому АМФК может играть роль в качестве ключевой молекулы в ИРФ-1 -регулируемом моделирования и ремоделирования кости, хотя роль остеобластной АМФК при передаче сигналов ИРФ-1 до сих пор неясна. Помимо этого, несколько исследований показали, что остеокальцин влияет на ось СТГ-ИРФ-1-инсулин и функцию печени. Это подтверждается тем, что введение остеокальцина достоверно увеличило секрецию СТГ из гипофиза и экспрессию печенью рецепторов к СТГ и ИРФ-1 [51]. Следовательно, сигнал ИРФ-1-инсулин-АМФК в кости может быть вовлечен во взаимодействие между костью и печенью.

В исследовании Kanazawa I et al., в 2008 году продемонстрировано, что метформин стимулирует дифференцировку и минерализацию остеобластов, и экспрессию остеокальцина путем активации АМФК [44], что даёт основание предположить, что активация АМФК может быть таргетной терапией снижения прочности кости при СД.

\section{ИЗУЧЕНИЕ МАРКЕРОВ КОСТНОГО РЕМОДЕЛИРОВАНИЯ ПРИ САХАРНОМ ДИАБЕТЕ}

По результатам метаанализа, проведенном Katrine Hygum et al., 2017 г. (метаанализ исследований за 2015-2016 гг.) было обнаружено снижение уровня циркулирующих маркеров костного обмена у пациентов с сахарным диабетом по сравнению с контрольной группой в отношении ряда маркеров резорбции кости и формирования. В данном метаанализе были изучены маркеры резорбции кости (CTX, NTX и TRAP), костного формирования (P1NP, остеокальцин и костно-специфическая щелочная фосфатаза (BAP)) и маркеры-регуляторы костного обмена (OPG, RANKL и склеростин).

Уровни СТX и остеокальцина были ниже в группе пациентов с СД 1 и 2 типов независимо от типа диабета по сравнению с группой контроля, что указывает на то, что как резорбция кости, так и костеобразование ниже при обоих типах диабета по сравнению с контролем. P1NP, маркер образования, также был ниже при СД1 и СД2 по сравнению с контролем. Склеростин был повышен у пациентов с диабетом типа 1 и типа 2; однако уровни склеростина были значительно выше у пациентов с СД2 типа по сравнению с группой контроля и группой пациентов с СД1 [52].

Для RANKL, NTX и BAP отличия были недостоверными в подгруппах диабета и при сравнении с контролем. TRAP была значительно ниже у пациентов с СД2 по сравнению с контролем. OPG был значительно выше при СД в целом по сравнению с контролем. В совокупности эти результаты свидетельствуют о том, что как СД1, так СД2 являются состояниями с замедленным костным обменом [52] (рис. 3).

BAP, маркер минерализации, в метаанализе не снижался у пациентов с диабетом по сравнению с контрольной группой. Возможно, что процесс костного обмена может быть разобщен - замедление резорбции и образования кости сопровождается интактной минерализацией. Таким образом, МПК может быть увеличена за счет этого механизма у пациентов с диабетом [52]

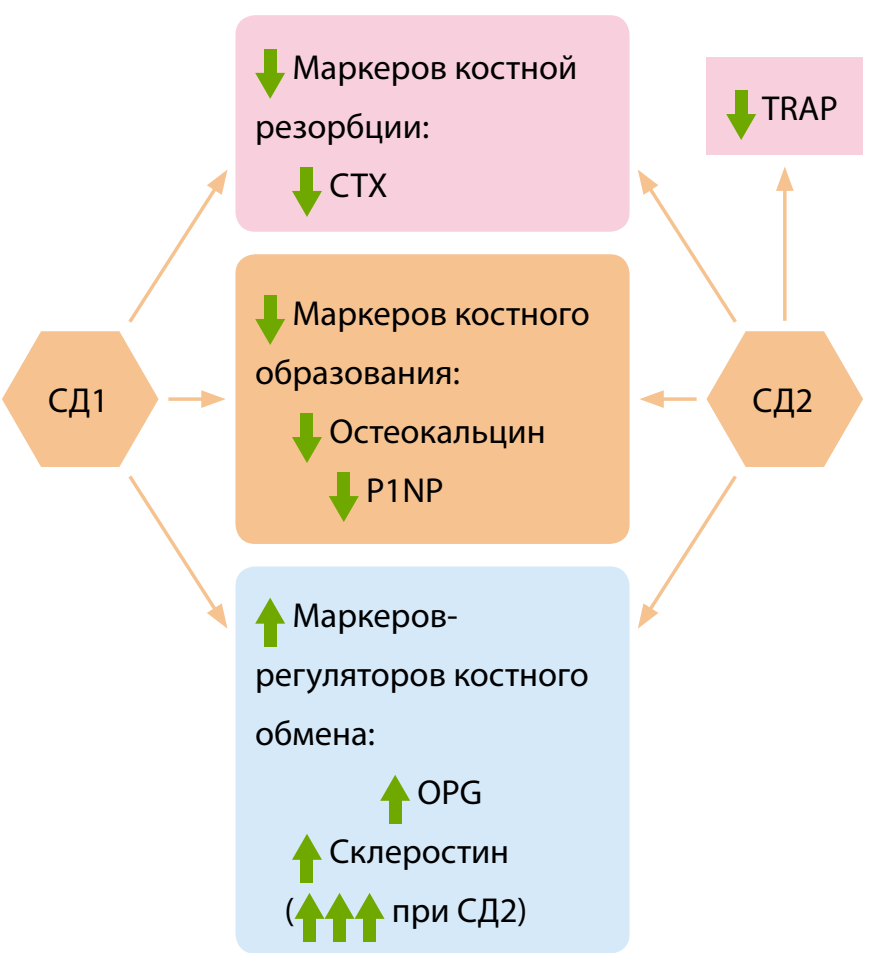

Рис. 3. Замедленный костный обмен при сахарном диабете

Недавнее кросс-секционное исследование, в котором приняло участие более 3000 мужчин, показало, что у мужчин с метаболическим синдромом, у которых оценивали CTX, P1NP и остеокальцин, образование и резорбция кости были ниже, чем у мужчин без метаболического синдрома. Маркеры костного обмена при метаболическом синдроме в особенности коррелировали с чувствительностью к инсулину, что указывает на то, что инсулинорезистентные индивидуумы могут иметь более низкий костный обмен, чем их здоровые сверстники [53].

Замедление костного обмена у пациентов с диабетом может быть объяснено повышением уровня склеростина. Уровни склеростина были повышены у пациентов с СД1 и СД2 по сравнению с контрольными группами. Уровни склеростина были пограничными, когда объединяли пациентов с диабетом типа 1 и типа 2, вероятно, из-за больших доверительных интервалов, возникающих в результате объединения результатов. Склеростин высвобождается из остеоцитов и уменьшает активность остеобластов и косвенно также снижает активность остеокластов, ингибируя секрецию OPG [54].

Склеростин секретируется остеоцитами, которые находятся в созревшей костной ткани, а не молодыми остеоцитами и остеоидными остеоцитами [55]. Склеростин ингибирует дифференцировку остеобластов и уменьшает формирование кости. У обоих полов склеростин сыворотки положительно, но слабо коррелирует с МПК [56], хотя корреляция выше у женщин в постменопаузе [57]. Неожиданная корреляция удивительна в отношении его антианаболического эффекта и не согласуется с возрастной потерей костной ткани. Было высказано предположение, что склеростин может быть важным маркером пула зрелых остеоцитов. Уровень склеростина отражает количество престарелой костной массы, которая может накапливать микротрещины и вызывать повышенный риск перелома, независимо от МПК, а также состояния костного обмена. 
Склеростин отрицательно влияет на минерализацию напрямую либо косвенно через регуляцию FGF23, другой молекулы, продуцируемой остеоцитами и участвующей в минерализации. Действительно, у мышей, нокаутных по гену склеростина (sost-/-), уменьшились уровни FGF23 и экскреция кальция в моче. Точный механизм снижения FGF-23 у (sost-/-) - нокаутных мышей неизвестен; высказывается предположение, что склеростин взаимодействует с PHEX (phosphate regulating endopeptidase homolog, X-linked) - белком, кодируемым фосфат-регулирующим геном, гомологичным эндопептидазе, сцепленным с X-хромосомой. PHEX снижает FGF-23. Вероятно, склеростин ингибирует PHEX и изменяет процессинг FGF-23 [58]. Склеростин и FGF23 могут синхронно действовать на почечные канальцы для повышения экскреции кальция и фосфора с мочой, тем самым снижая доступность этих элементов для минерализации.

Экспрессия склеростина регулируется несколькими системными и местными факторами, такими как кальцитриол в отдельности или в комбинации с глюкокортикоидами, фактор некроза опухоли-а и костные морфогенные белки. И наоборот, ПтГ уменьшает остеоцитарную экспрессию склеростина в клеточных линиях и у мышей [59].

В исследовании Y. WU et al., 2017, в котором приняли участие 32 пациента с СД2 и переломом шейки бедра и 27 пациентов с переломом шейки бедра без диабета (обе выборки включали мужчин и женщин), было продемонстрировано, что в группе сахарного диабета был достоверно повышен уровень склеростина, который отрицательно коррелировал с остеокальцином, P1NP и СТХ. Результаты in vitro подтвердили, что склеростин понижает экспрессию остеокальцина, Р1NP и CTX в hFOB 1.19 клетках. Это говорит о том, что склеростин может быть эффективной целью терапии при переломах кости, ассоциированных с сахарным диабетом и при задержке заживления переломов [60].

В исследованиях in vitro сообщается, что гипергликемия увеличивает экспрессию OPG в клеточных линиях остеобластов [61] и увеличивает экспрессию склеростина клеточными линиями остеоцитов [62]. Повышение уровня глюкозы в плазме ассоциировано с увеличением уровня OPG у пациентов с диабетом [63]. Кроме того, уровни OPG в сыворотке снижаются у женщин без диабета во время теста на толерантность к глюкозе, тогда как у женщин с диабетом 2 типа [64] этого не происходит. Отсутствие ответа OPG на пероральный уровень глюкозы у женщин с диабетом типа 2 может быть вызвано хронической гипергликемией, которая ограничивает дальнейшее уменьшение OPG. Хотя существующих данных недостаточно, уровни как OPG, так и склеростина могут быть увеличены при диабете из-за гипергликемии. Следовательно, повышенный уровень OPG и склеростина могут замедлять костный обмен у пациентов с диабетом.

\section{ЗАКЛЮЧЕНИЕ}

Нарушения костного обмена у пациентов сахарным диабетом связаны с высоким уровнем циркулирующих КПГ и гомоцистеина, которые напрямую нарушают функции остеобластов и остеоцитов, способствуют уменьшению костного формирования и замедлению ремоделирования. Адипонектин стимулирует экспрессию остеокальцина и дифференцировку остеобластов посредством активации АМФК. Гипоадипонектинемия, которая часто наблюдается при ожирении и диабете, может участвовать в снижении прочности кости при СД.

Замедленный костный метаболизм у пациентов сахарным диабетом подтверждается при изучении уровня маркеров костного ремоделирования. Об этом свидетельствует снижение уровня маркеров костного образования (остеокальцин, Р1NP), маркеров костной резорбции (CTX, TRAP), повышение маркеров-регуляторов костного обмена (OPG, склеростин).

Необходимы большие когортные исследования для определения прогностической ценности маркеров костного обмена у пациентов с диабетом в отношении риска переломов.

\section{ДОПОЛНИТЕЛЬНАЯ ИНФОРМАЦИЯ}

Источник финансирования. Поисково-аналитическая работа проведена на личные средства авторского коллектива.

Конфликт интересов. Авторы декларируют отсутствие явных и потенциальных конфликтов интересов, связанных с публикацией настоящей статьи.

\section{СПИСОК ЛИТЕРАТУРЫ | REFERENCES}

1. Vestergaard P. Discrepancies in bone mineral density and fracture risk in patients with type 1 and type 2 diabetes a meta-analysis. Osteoporos Int. 2007;18(4):427-44. doi: 10.1007/s00198-006-0253-4

2. Janghorbani M, Van Dam RM, Willett WC et al. Systematic review of type 1 and type 2 diabetes mellitus and risk of fracture. Am J Epidemiol. 2007;166(5):495-505 doi: 10.1093/aje/kwm106

3. Yamamoto $M$, Yamaguchi T, Yamauchi M et al. Diabetic patients have an increased risk of vertebral fractures independent of bone mineral density or diabetic complications. J Bone Miner Res. 2009;24(4):702-709. doi: 10.1359/jbmr.081207

4. Kanazawa I. Interaction between bone and glucose metabolism Endocr J. 2017;64:1043-1053. doi: 10.1507/endocrj.EJ17-0323

5. Ялочкина Т.О., Белая Ж.Е. Низкотравматичные переломы и костное ремоделирование при сахарном диабете 2 типа. // Ожирение и метаболизм. - 2017. - Т.14. - №. 3 - C.11-18. [Yalochkina TO, Belaya ZhE. Fragility fractures and bone remodeling in type 2 diabetes mellitus. Obesity and metabolism. 2017;14(3):11-18. (In Russ).] doi: 10.14341/OMET2017311-18
6. Grandhee SK, Monnier VM. Mechanism of formation of the Maillard protein cross-link pentosidine. Glucose, fructose, and ascorbate as pentosidine precursors. J Biol Chem. 1991;266: 11649-11653

7. Saito M, Fujii K, Mori Y, Marumo K. Role of collagen enzymatic and glycation induced cross-links as a determinant of bone quality in spontaneously diabetic WBN/Kob rats. Osteoporos Int. 2006;17(10):1514-23. doi: 10.1007/s00198-006-0155-5

8. Schwartz AV, Garnero P, Hillier TA et al. Pentosidine and increased fracture risk in older adults with type 2 diabetes. J Clin Endocrinol Metab. 2009;94(7):2380-2386. doi: 10.1210/jc.2008-2498

9. Yamamoto M, Yamaguchi T, Yamauchi M et al. Serum pentosidine levels are positively associated with the presence of vertebral fractures in postmenopausal women with type 2 diabetes. J Clin Endocrinol Metab. 2008;93(3):1013-9. doi: 10.1210/jc.2007-1270

10. Farlay D, Armas LA, Gineyts E et al. Nonenzymatic glycation and degree of mineralization are higher in bone from fractured patients with type 1 diabetes mellitus. J Bone Miner Res. 2016;31(1):190-195. doi: 10.1002/jbmr.2607 
11. Farr JN, Khosla S. Determinants of bone strength and quality in diabetes mellitus in humans. Bone. 2016;82:28-34. doi: 10.1016/j.bone.2015.07.027

12. Furst JR, Bandeira LC, Fan WW et al. Advanced glycation endproducts and bone material strength in type 2 diabetes. J Clin Endocrinol Metab. 2016;101(6):2502-2510. doi: 10.1210/jc.2016-1437

13. Tanaka K, Yamaguchi T, Kanazawa I et al. Effects of high glucose and advanced glycation end products on the expressions of sclerostin and RANKL as well as apoptosis in osteocyte-like MLOY4-A2 cells. Biochem Biophys Res Commun. 2015;461(2):193-199. doi: 10.1016/j.bbrc.2015.02.091

14. Ogawa N, Yamaguchi T, Yano S et al. The combination of high glucose and advanced glycation end-products (AGEs) inhibits the mineralization of osteoblastic MC3T3-E1 cells through glucose-induced increase in the receptor for AGEs. Horm Metab Res 2007;39(12): 871-875. doi: 10.1055/s-2007-991157

15. Okazaki K, Yamaguchi T, Tanaka K et al. Advanced glycation end products (AGEs), but not high glucose, inhibit the osteoblastic differentiation of mouse stromal ST2 cells through the suppression of osterix expression, and inhibit cell growth and increasing cell apoptosis. Calcif Tissue Int. 2012;91(4):286-296. doi: 10.1007/s00223-012-9641-2

16. Tanaka K, Yamaguchi T, Kaji H et al. Advanced glycation end products suppress osteoblastic differentiation of stromal cells by activation endoplasmic reticulum stress. Biochem Biophys Res Commun. 2013; 438(3):463-467. doi: 10.1016/j.bbrc.2013.07.126

17. van Meurs JB, Dhonukshe-Rutten RA, Pluijm SM et al. Homocysteine levels and the risk of osteoporotic fracture. N Engl J Med. 2004;350(20):2033-2041. doi: 10.1056/NEJMoa032546

18. Yang J, Hu X, Zhang Q et al. Homocysteine level and risk of fracture: A meta-analysis and systematic review. Bone. 2012;51(3):376-382 doi: 10.1016/j.bone.2012.05.024

19. Li J, Zhang H, Yan L et al. Fracture is additionally attributed to hyperhomocysteinemia in men and premenopausal women with type 2 diabetes. J Diabetes Investig. 2014;5(2):236-241. doi: 10.1111/jdi.12149

20. Saito M, Marumo K, Soshi S et al. Raloxifene ameliorates detrimental enzymatic and nonenzymatic collagen cross-links and bone strength in rabbits with hyperhomocysteinemia. Osteoporos Int 2010;21(4):655-666. doi: 10.1007/s00198-009-0980-4

21. Kanazawa I, Tomita T, Miyazaki S et al. Bazedoxifene ameliorates homocysteine-induced apoptosis and accumulation of advanced glycation end products by reducing oxidative stress in MC3T3-E1 cells. Calcif Tissue Int. 2017;100(3):286-297. doi: 10.1007/s00223-016-0211-x

22. Takeno A, Kanazawa I, Tanaka K et al. Activation of AMPK-activated protein kinase protects against homocysteine-induced apoptosis of osteocytic MLO-Y4 cells by regulating the expressions of NADPH oxidase 1 (Nox1) and Nox2. Bone. 2015;77:135-141. doi: 10.1016/j.bone.2015.04.025.

23. Takeno A, Kanazawa I, Tanaka K et al. Simvastatin rescues homocysteine-induced apoptosis of osteocytic MLO-Y4 cells by decreasing the expressions of NADPH oxidase 1 and 2. Endocr J. 2016;63(4):389-395. doi: 10.1507/endocri.EJ15-0480

24. Verhaeghe J, Suiker AM, Visser WJ et al. The effects of systemic insulin, insulin-like growth factor-I and growth hormone on bone growth and turnover in spontaneously diabetic BB rats. J Endocrinol. 1992:134(3):485-492

25. Pun KK, Lau P, Ho PW. The characterization, regulation, and function of insulin receptors on osteoblast- like clonal osteosarcoma cell line. J Bone Miner Res. 1989;4(6): 853-862

26. Fulzele K, Riddle RC, Digirolamo DJ et al. Insulin receptor signaling in osteoblasts regulates postnatal bone acquisition and body composition. Cell. 2010;142(2):309-19. doi: 10.1016/j.cell.2010.06.002

27. Zhang M, Xuan S, Bouxsein ML et al. Osteoblast-specific knockout of the insulin-like growth factor (IGF) receptor gene reveals and essential role of IGF signaling in bone matrix mineralization. J Biol Chem. 2002;277(46):44005-40012. doi: 10.1074/jbc.M208265200

28. Yakar S, Rosen CJ, Beamer WG et al. Circulating levels of IGF-I directly regulate bone growth and density. J Clin Invest. 2002;110(6):771-781. doi: $10.1172 / J C l 15463$

29. Terada M, Inaba M, Yano Y et al. Growth-inhibitory effect of a high glucose concentration on osteoblast-like cells. Bone. 1998;22(1):17-23

30. McCarthy AD, Etcheverry SB, Cortizo AM. Effect of advanced glycation endproducts on the secretion of insulin-like growth factor-I and its binding proteins: role in osteoblast development. Acta Diabetol. 2001:38(3):113-22
31. Kanazawa I, Yamaguchi T, Yamamoto M et al. Serum insulin-like growth factor-l is associated with the presence of vertebral fractures in postmenopausal women with type 2 diabetes mellitus. Osteoporos Int. 2007;18(12):1675-1681. doi: 10.1007/s00198-007-0430-0.

32. Morley JE, Baumgartner RN. Cytokine-related aging process. J Gerontol A Biol Sci Med Sci. 2004;59(9):M924-M929

33. Kanazawa I, Yamaguchi T, Yano S et al. Adiponectin and AMP kinase activator stimulate proliferation, differentiation, and mineralization of osteoblastic MC3T3-E1 cells. BMC Cell Biol. 2007:8:51. doi: 10.1186/1471-2121-8-51

34. Luo XH, Guo LJ, Yuan LQ et al. Adiponectin stimulates human osteoblasts proliferation and differentiation via the MAPK signaling pathway. Exp Cell Res. 2005;309(1):99-109. doi: 10.1016/j.yexcr.2005.05.021

35. Luo XH, Guo LJ, Xie H et al. Adiponectin stimulates RANKL and inhibits OPG expression in human osteoblasts through the MAPK signaling pathway. J Bone Miner Res. 2006;21(10):1648-1656. doi: 10.1359/jbmr.060707

36. Kanazawa I, Yamaguchi T, Yamamoto $\mathrm{M}$ et al. Relationships between serum adiponectin levels versus bone mineral density, bone metabolic markers, and vertebral fractures in type 2 diabetes mellitus. Eur J Endocrinol. 2009;160(2):265-73. doi: 10.1530/EJE-08-0642

37. Richards JB, Valdes AM, Burling K et al. Serum adiponectin and bone mineral density in women. J Clin Endocrinol Metab. 2007:92(4):1517-1523. doi: 10.1210/jc.2006-2097

38. Johansson $\mathrm{H}$, Oden A, Karlsson MK et al. Waning predictive value of serum adiponectin for fracture risk in elderly men: MrOS Sweden. Osteoporos Int. 2014;25(7):1831-6. doi: 10.1007/s00198-014-2654-0

39. Ruderman NB, Carling D, Prentki M et al. AMPK, insulin resistance, and the metabolic syndrome. J Clin Invest. 2013;123(7):2764-2772. doi: $10.1172 / \mathrm{JCl} 67227$

40. Motoshima H, Goldstein BJ, Igata M et al. AMPK and cell proliferationAMPK as a therapeutic target for atherosclerosis and cancer. J Physiol. 2006;574(Pt 1):63-71. doi: 10.1113/jphysiol.2006.108324

41. Quinn JM, Tam S, Sims NA et al. Germline deletion of AMP-activated protein kinase beta subunits reduces bone mass without altering osteoclast differentiation or function. FASEB J. 2010; 24(1): 275-285. doi: 10.1096/fj.09-137158

42. Jeyabalan J, Shah M, Viollet B et al. AMP activated protein kinase pathway and bone metabolism. J Endocrinol. 2012;212(3):277-290. doi: 10.1530/JOE-11-0306

43. Shah M, Kola B, Bataveljic A et al. AMP-activated protein kinase (AMPK) activation regulates in vitro bone formation and bone mass. Bone. 2010:47(2):309-319. doi: 10.1016/j.bone.2010.04.596.

44. Kanazawa I, Yamaguchi T, Yano $S$ et al. Metformin enhances the differentiation and mineralization of osteoblastic MC3T3-E1 cells via AMP kinase activation as well as eNOS and BMP-2 expression. Biochem Biophys Res Commun. 2008;375(3):414-419. doi: 10.1016/j.bbrc.2008.08.034

45. Jang WG, Kim EJ, Lee KN et al. AMP-activated protein kinase (AMPK) positively regulates osteoblast differentiation via induction of Dlx5dependent Runx2 expression in MC3T3E1 cells. Biochem Biophys Res Commun. 2011;404(4):1004-1009. doi: 10.1016/j.bbrc.2010.12.099

46. Kang H, Viollet B, Wu D. Genetic deletion of catalytic subunits of AMP-activated protein kinase increases osteoclasts and reduces bone mass in young adult mice. J Biol Chem. 2013:288(17):12187-12196. doi: 10.1074/jbc.M112.430389

47. Yokomoto-Umakoshi M, Kanazawa I, Takeno A et al. Activation of AMP-activated protein kinase decreases receptor activator of NF-KB ligand expression and increases sclerostin expression by inhibiting the mevalonate pathway in osteocytic MLOY4 cells. Biochem Biophys Res Commun. 2016;469(4):791-796. doi: 10.1016/j.bbrc.2015.12.072.

48. Jeyabalan J, Shah M, Viollet B et al. Mice lacking AMP-activated protein kinase a1 catalytic subunit have increased bone remodeling and modified skeletal responses to hormonal challenges induced by ovariectomy and intermittent PTH treatment. J Endocrinol. 2012;214(3):349-358. doi: 10.1530/JOE-12-0184

49. Tulipano $G$, Faggi $L$, Sibilia $\vee$ et al. Points of integration between the intracellular energy sensor AMP-activated protein kinase (AMPK) activity and the somatotroph axis function. Endocrine. 2012:42(2):292298 doi: 10.1007/s12020-012-9732-x

50. Xi G, Rosen CI, Clemmons DR. IGF-I and IGFBP-2 stimulate AMPK activation and autophagy, which are required for osteoblast differentiation. Endocrinology. 2016; 157(1): 268-281 doi: 10.1210/en.2015-1690.

51. Li Y, Li K. Osteocalcin induced growth hormone/insulin-like growth factor-1 system by promoting testosterone syn- 
thesis in male mice. Horm Metab Res. 2014:46(11):768-73. doi: 10.1055/s-0034-1371869

52. Hygum K, Starup-Linde J, Harslof T et al. Mechanisms in endocrinology: Diabetes mellitus, a state of low bone turnover - a systematic review and meta-analysis. Eur J Endocrinol. 2017;176(3):R137-R157. doi: 10.1530/EJE-16-0652

53. Laurent MR, Cook MJ, Gielen E et al. Lower bone turnover and relative bone deficits in men with metabolic syndrome: a matter of insulin sensitivity? The European Male Ageing Study. Osteoporosis Int 2016; 27(11):3227-3237. doi:10.1007/s00198-016-3656-x

54. Manolagas SC \& Almeida M. Gone with the Wnts: beta-catenin, T-cell factor, forkhead box $\mathrm{O}$, and oxidative stress in age dependent diseases of bone, lipid, and glucose metabolism. Mol Endocrinol. 2007;21(11):2605-14. doi:10.1210/me.2007-0259

55. Dallas SL, Bonewald LF. Dynamics of the transition from osteoblast to osteocyte. Ann N Y Acad Sci. 2010;1192:437-43. doi: 10.1111/j.1749-6632.2009.05246.x.

56. Garnero P, Sornay-Rendu E, Munoz F et al. Association of serum sclerostin with bone mineral density, bone turnover, steroid and parathyroid hormones, and fracture risk in postmenopausal women: the OFELY study. Osteoporos Int. 2013;24(2):489-94. doi: 10.1007/s00198-012-1978-x

57. Modder UI, Clowes JA, Hoey K et al. Regulation of circulating sclerostin levels by sex steroids in women and in men. $J$ Bone Miner Res. 2011;26(1):27-34. doi: 10.1002/jbmr.128
58. Ryan ZC, Ketha H, McNulty MS et al. Sclerostin alters serum vitamin D metabolite and fibroblast growth factor 23 concentrations and the urinary excretion of calcium. Proc Natl Acad Sci U S A. 2013;110(15):6199-204. doi: 10.1073/pnas.1221255110

59. Keller $\mathrm{H}$, Kneissel $\mathrm{M}$ : SOST is a target gene for PTH in bone. Bone. 2005;37(2):148-58. doi: 10.1016/j.bone.2005.03.018

60. Wu Y, Xu SY, Liu SY et al. Upregulated serum sclerostin level in the T2DM patients with femur fracture inhibits the expression of bone formation/remodeling-associated biomarkers via antagonizing Wnt signaling. Eur Rev Med Pharmacol Sci. 2017;21 (3):470-478

61. Liu Z, Jiang $\mathrm{H}$, Dong K et al. Different concentrations of glucose regulate proliferation and osteogenic differentiation of osteoblasts via the PI3 kinase/Akt pathway. Implant Dent. 2015;24(1):83-91. doi: 10.1097/ID.0000000000000196

62. Tanaka K-I, Yamaguchi T, Kanazawa I et al. Effects of high glucose and advanced gexpressions of sclerostin and RANKL as well as apoptosis in osteocyte-like MLO-Y4-A2 cells. Biochem Biophys Res Commun. 2015:461 (2):193-9. doi: 10.1016/j.bbrc.2015.02.091

63. Starup-Linde J, Lykkeboe S, Gregersen S et al. Differences in biochemical bone markers by diabetes type and the impact of glucose. Bone. 2016;83:149-55. doi: 10.1016/j.bone.2015.11.004

64. Chailurkit LO, Chanprasertyothin S, Rajatanavin R et al. Reduced attenuation of bone resorption after oral glucose in type 2 diabetes. Clinical Endocrinology 2008;68(6):858-862 doi:10.1111/j.1365-2265.2007.03159.x

\section{ИНФОРМАЦИЯ ОБ АВТОРАХ [AUTHORS INFO]}

Нуруллина Гузель Михайловна, аспирант [Guzel M. Nurullina, MD, PhD-student]; адрес: Россия, 426039, Удмуртская республика, Ижевск, улица Воткинское шоссе, д.57 [address: 57 Votkinskoe shosse street, 426039 Izhevsk, Russia]; ORCID: https://orcid.org/0000-0002-4394-8446; eLibrary SPIN: 5885-7116; e-mail: dalllila@mail.ru

Ахмадуллина Гузяль Илгисовна, к.м.н., доцент [Guzyal I. Akhmadullina, MD, PhD, assistant professor]; ORCID: https://orcid.org/0000-0003-1876-2516; eLibrary SPIN: 4171-1245; e-mail: guzal-work@mail.ru

\section{ЦИТИРОВАТЬ:}

Нуруллина Г.М., Ахмадуллина Г.И. Особенности костного метаболизма при сахарном диабете // Остеопороз и остеопа-тии. - 2017. - Т. 20. — №3. — C.82-89. doi: 10.14341/osteo2017382-89

\section{TO CITE THIS ARTICLE:}

Nurullina GM, Akhmadullina Gl. Features of bone turnover in diabetes mellitus. Osteoporosis and bone diseases. 2017;20(3):82-89. doi: 10.14341/osteo2017382-89 